Ann. Génét. Sél. anim., I973, 5 (I), 73-82.

\title{
MÉTHODE DE CALCUL DES INDEX DE PRODUCTION LAITIÈRE DES FEMELLES EN FRANCE
}

\author{
J.-J. COLLEAU et M. POUTOUS \\ Station de Génétique quantitative et appliquée, \\ Centre national de Recherches zootechniques, I. N. R. A., \\ 78350 Jouy en Josas
}

\begin{abstract}
RÉSUMÉ
Nous avons décrit la méthode utilisée en France pour le calcul des index de production laitière des femelles bovines, ovines et caprines. Son principe consiste en l'utilisation optimale, par la méthode de régression multiple, non de performances mais d'index, en particulier des index précédemment calculés. Les formules de mise à jour et leurs justifications théoriques sont explicitées. On a abordé de la même manière le problème de la combinaison d'informations de diverses origines (performances des apparentés, performances des individus).

L'intérêt de la méthode réside dans la réduction du nombre des données à stocker, la validité de formules de cumul peu nombreuses pour une grande gamme de situations particulières, la vitesse d'exécution des calculs. Cela peut s'accompagner d'une réduction de la précision. Quand elle existe, elle n'est pas constante dans le temps, mais se maintient à un faible niveau.
\end{abstract}

\section{INTRODUCTION}

Un choix raisonné des femelles reproductrices, tout particulièrement celui des mères des reproducteurs mâles, est susceptible d'augmenter le progrès génétique annuel moyen dans une proportion importante. Dans le cas res populations de femelles bovines traites et soumises à insémination artificielle, l'amélioration est d'environ 25 à 35 p. Ioo (SkJERvold et LANGHOLz, I964; Lindhe, I968). Cette estimée théorique se révèle en fait souvent trop faible dans les études a posteriori de populations réelles (SyRstad, I966; Lindström, I969).

En pratique la sélection des femelles, mères possibles des futurs reproducteurs mâles, n'a d'intérêt que si la pression de sélection et la précision du choix sont suffisantes. La pression de sélection dépend d'abord du nombre de femelles contrôlées et de la bonne volonté des éleveurs pour la livraison des veaux mâles. Elle peut cependant être augmentée par un système de calcul suffisamment simple et entraînant des coûts d'exploitation mécanographique assez bas pour qu'il puisse être étendu à l'ensemble des animaux contrôlés. La précision du choix, elle, peut être améliorée, 
d'une part en corrigeant les performances pour l'effet des principaux facteurs connus du milieu, d'autre part en tenant compte des performances des animaux apparentés (ascendants, collatéraux et descendants). Pour faciliter la tâche des sélectionneurs, une condensation des informations en une seule valeur affectée d'un coefficient de détermination est très souhaitable. Cette valeur que nous appellerons index correspond à l'estimée sans biais la plus précise possible (variance d'erreur minimale) de la valeur génétique d'origine additive pour le sujet considéré, dans la mesure où les hypothèses à la base du calcul sont satisfaites.

Une méthode d'indexation des femelles laitières répondant à ces préoccupations pratiques et actuellement utilisée en France a déjà été décrite (Pourous et CROCHEZ, I97I). Une de ses originalités est qu'elle aboutit à l'index global d'un sujet par une suite de combinaisons deux à deux d'index élémentaires. Notre but est d'expliciter les bases d'un tel calcul puis d'appliquer les résultats obtenus à la méthode précitée d'indexation et enfin de discuter brièvement des avantages et inconvénients de cette solution.

\section{NOTIONS THÉORIQUES}

Le problème théorique de la condensation de plusieurs index soit d'origine différente (performance, ascendance, descendance) soit obtenus à des moments différents dans le temps sur le même animal, peut se résumer de la manière suivante. Connaissant l'animal sur deux index $I_{1}$ et $I_{2}$ supposés d'espérance mathématique nulle et ayant chacun des précisions connues, quels sont les coefficients optima $a_{1}$ et $a_{2}$ à utiliser pour construire l'index final $\mathrm{I}=a_{1} \mathrm{I}_{1}+a_{2} \mathrm{I}_{2}$ ? Quelle est alors la précision de ce nouvel index?

Le problème posé peut être résolu par la méthode de la régression multiple. Si $v\left(\mathrm{I}_{1}\right)$ et $v\left(\mathrm{I}_{2}\right)$ sont les variances des index; $\operatorname{cov}\left(I_{1}, I_{2}\right)$ la covariance entre ces index; $\operatorname{cov}\left(G, I_{1}\right), \operatorname{cov}\left(G, I_{2}\right)$ les covariances respectives entre index et valeur génétique à estimer, le vecteur colonne des coefficients optima dans le nouvel index peut s'écrire :

$$
\left(\begin{array}{l}
a_{1} \\
a_{2}
\end{array}\right)=\left(\begin{array}{ll}
v\left(\mathrm{I}_{1}\right) & \operatorname{cov}\left(\mathrm{I}_{1}, \mathrm{I}_{2}\right) \\
\operatorname{cov}\left(\mathrm{I}_{1}, \mathrm{I}_{2}\right) & v\left(\mathrm{I}_{2}\right)
\end{array}\right)^{-1}\left(\begin{array}{l}
\operatorname{cov}\left(\mathrm{G}, \mathrm{I}_{1}\right) \\
\operatorname{cov}\left(\mathrm{G}, \mathrm{I}_{2}\right)
\end{array}\right)
$$

soit en désignant par des lettres ces trois matrices

$$
\mathrm{A}=\mathrm{V}^{-1} \mathrm{C}
$$

Les coefficients $a_{1}$ et $a_{2}$ sont tels qu'ils minimisent la variance de l'erreur d'estimation $e$ de la valeur génétique $\mathrm{G}$ dans le modèle, $\mathrm{G}=\mathrm{I}+e$. Les hypothèses portant sur $e$ sont que son espérance mathématique est nulle, que sa variance ne dépend pas de $I$, que ses covariances avec $I_{1}$ et $\mathrm{I}_{2}$ sont nulles.

Nous évaluerons la précision du nouvel index par son coefficient de détermination $\mathrm{CD}$ qui représente la part de variance génétique qui est contrôlée par cet index

$$
\mathrm{CD}=\frac{v(\mathrm{I})}{v(\mathrm{G})}
$$

où $v(\mathrm{I})$ et $v(\mathrm{G})$ désignent la variance de l'index et la variance génétique.

La résolution du système linéaire et le calcul de $C D$ donnent les résultats suivants :

$$
\begin{gathered}
a_{1}=\frac{\operatorname{cov}\left(\mathrm{G}, \mathrm{I}_{1}\right) v\left(\mathrm{I}_{2}\right)-\operatorname{cov}\left(\mathrm{G}, \mathrm{I}_{2}\right) \operatorname{cov}\left(\mathrm{I}_{1}, \mathrm{I}_{2}\right)}{v\left(\mathrm{I}_{1}\right) v\left(\mathrm{I}_{2}\right)-\operatorname{cov}^{2}\left(\mathrm{I}_{1}, \mathrm{I}_{2}\right)} \\
a_{2}=\frac{\operatorname{cov}\left(\mathrm{G}, \mathrm{I}_{2}\right) v\left(\mathrm{I}_{1}\right)-\operatorname{cov}\left(\mathrm{G}, \mathrm{I}_{1}\right) \operatorname{cov}\left(\mathrm{I}_{1}, \mathrm{I}_{2}\right)}{v\left(\mathrm{I}_{1}\right) v\left(\mathrm{I}_{2}\right)-\operatorname{cov}^{2}\left(\mathrm{I}_{1}, \mathrm{I}_{2}\right)} \\
\mathrm{CD}=\frac{\mathrm{I}}{v(\mathrm{G})} \frac{\operatorname{cov}^{2}\left(\mathrm{G}, \mathrm{I}_{1}\right) v\left(\mathrm{I}_{2}\right)+\operatorname{cov}^{2}\left(\mathrm{G}, \mathrm{I}_{2}\right) v\left(\mathrm{I}_{1}\right)-2 \operatorname{cov}\left(\mathrm{G}, \mathrm{I}_{1}\right) \operatorname{cov}\left(\mathrm{G}, \mathrm{I}_{2}\right) \operatorname{cov}\left(\mathrm{I}_{1}, \mathrm{I}_{2}\right)}{v\left(\mathrm{I}_{1}\right) v\left(\mathrm{I}_{2}\right)-\operatorname{cov}^{2}\left(\mathrm{I}_{1}, \mathrm{I}_{2}\right)}
\end{gathered}
$$


Il s'agit maintenant d'expliciter analytiquement les variances et covariances entre index. Par définition, nous venons de voir que

$$
v(\mathrm{I})=\mathrm{CD} v(\mathrm{G})
$$

Pour calculer les covariances entre index estimant la même valeur génétique, il est nécessaire de poser un modèle de prédiction de I connaissant G. Le modèle dont la variance d'erreur est minimale (méthode des moindres carrés) est le suivant:

$$
\mathrm{I}=\frac{\operatorname{cov}(\mathrm{G}, \mathrm{I})}{v(\mathrm{G})} \mathrm{G}+\varepsilon
$$

$\varepsilon$ étant une erreur aléatoire indépendante de $\mathrm{G}$. On peut montrer que

$$
\operatorname{cov}(\mathrm{G}, \mathrm{I})=v(\mathrm{I})
$$

D'après les notations matricielles précédentes $\operatorname{cov}(G, I)={ }^{t} \mathrm{CV}^{-1} \mathrm{C}$ où ${ }^{t} \mathrm{C}$ désigne la matrice transposée de C. D'autre part, si l'on désigne par $L$ le vecteur colonne $\left(\begin{array}{l}\mathrm{I}_{1} \\ \mathrm{I}_{2}\end{array}\right)$ on a

$$
\mathrm{I}={ }^{t} \mathrm{C}^{t}\left(\mathrm{~V}^{-1}\right) \mathrm{L}={ }^{t} \mathrm{CV}^{-1} \mathrm{~L} \quad \text { puisque } \mathrm{V}^{-1} \text { est une matrice symétrique }
$$

Puisque I est une valeur numérique on a également

$$
\mathrm{I}={ }^{t} \mathrm{I}={ }^{t} \mathrm{LV}^{-1} \mathrm{C}
$$

soit

$$
v(\mathrm{I})=\mathrm{E}\left(\mathrm{I}^{2}\right)=\mathrm{E}\left({ }^{t} \mathrm{CV}^{-1} \mathrm{~L}^{t} \mathrm{LV}^{-1} \mathrm{C}\right)={ }^{t} \mathrm{CV}^{-1} \mathrm{E}\left(\mathrm{L}^{t} \mathrm{~L}\right) \mathrm{V}^{-1} \mathrm{C}
$$

Or

$$
\mathrm{E}\left(\mathrm{L}^{t} \mathrm{~L}\right)=\mathrm{V} \quad \text { soit } v(\mathrm{I})={ }^{t} \mathrm{CV}^{-1} \mathrm{VV}^{-1} \mathrm{C}={ }^{t} \mathrm{CV}^{-1} \mathrm{C}=\operatorname{cov}(\mathrm{G}, \mathrm{I})
$$

Soit

On a ainsi

$$
\frac{\operatorname{cov}(\mathrm{G}, \mathrm{I})}{v(\mathrm{G})}=\frac{v(\mathrm{I})}{v(\mathrm{G})}=\mathrm{CD} \frac{v(\mathrm{G})}{v(\mathrm{G})}=\mathrm{CD}
$$

$$
\mathrm{I}=\mathrm{CDG}+\varepsilon
$$

De l'équation (7) il est alors possible de déduire les covariances entre index. $\mathrm{Si}$

on a

$$
\mathrm{I}_{1}=\mathrm{CD}_{1} \mathrm{G}+\varepsilon_{1} \quad \text { et } \quad \mathrm{I}_{2}=\mathrm{CD}_{2} \mathrm{G}+\varepsilon_{2}
$$

$$
\operatorname{cov}\left(\mathrm{I}_{1}, \mathrm{I}_{2}\right)=\mathrm{CD}_{1} \mathrm{CD}_{2} v(\mathrm{G})+\operatorname{cov}\left(\varepsilon_{1}, \varepsilon_{2}\right)
$$

La méthode générale de calcul des index dont nous venons de définir les bases théoriques peut être itérative, en ce sens que l'index final I, combiné à un index élémentaire $\mathrm{I}_{3}$ peut servir à estimer un nouvel index I' plus précis que l'ancien et ainsi de suite. Nous pouvons alors schématiser le traitement de l'information de la manière suivante :

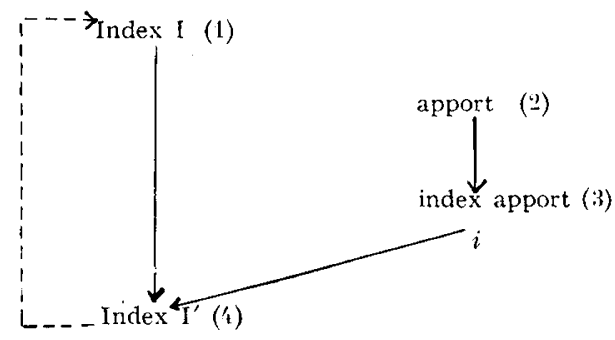

(I) synthèse de toutes les informations déjà traitées,

(2) information non encore traitée,

(3) index du sujet obtenu à pariir de l'apport,

(4) cet index se trouvera de nouveau en (I) si une nouvelle information (2) est à traiter. 
Les formules de calcul des index $i$ ainsi que les formules de cumul permettant de réaliser les boucles de calcul nécessitent pour pouvoir être mises en œuvre d'avoir été étudiées pour chaque type de cumul à envisager (cumul d'informations de types différents, cumul d'informations de même type mais s'échelonnant dans le temps). C'est ce que nous nous proposons de montrer dans le paragraphe suivant pour le cas de l'indexation des femelles laitières.

\section{APPLICATION A L'INDEXATION DES FEMELLES LAITIÈRES}

\section{A. - Principes généraux}

Le traitement des données effectué pour l'indexation des femelles laitières peut être représenté graphiquement (fig. I). Le système de cumul des index que nous avons décrit est appliqué séparément pour 2 catégories d'informations : celles sur les propres performances du sujet, celles sur apparentés. L'index global mis à jour est obtenu par combinaison, à chaque fois, des valeurs terminales de ces index de façon à simplifier les formules de calcul.

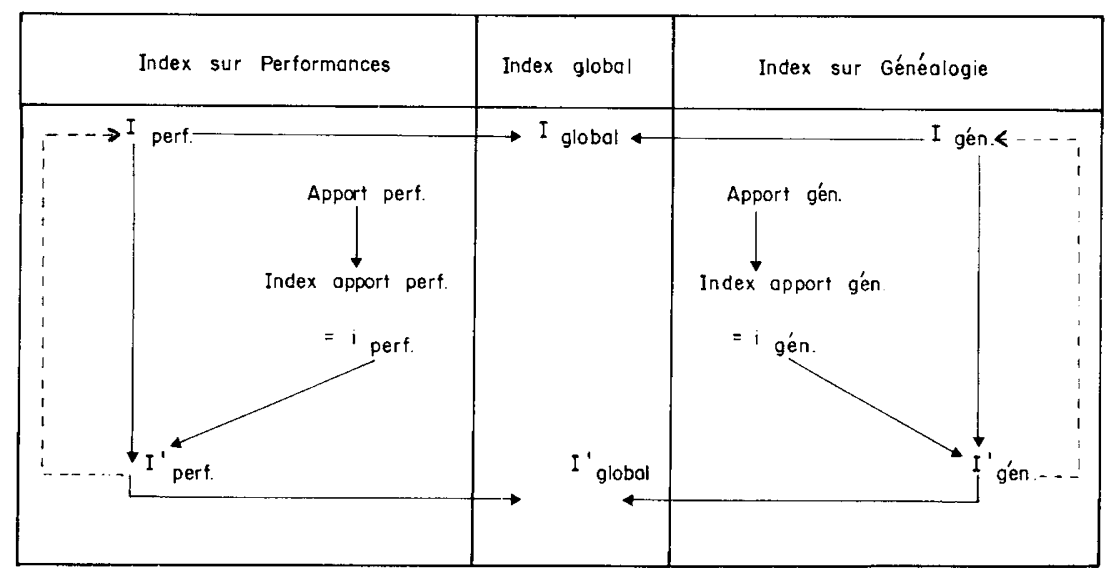

FIG. I. - Schéma de calcul et de mise à jour des index

L'index sur performance stocké en mémoire (index ancien) résume l'ensemble de performances connues de l'animal. L'index ancien sur généalogie résume l'information apportée par les animaux apparentés, quelle que soit la nature de l'apparentement et quel que soit le nombre de parents connus (ascendants, collatéraux et descendants).

La mise à jour de ces différents index s'effectue en deux phases. D'abord l'information nouvelle est transformée en index apport. Les performances de l'animal sont ainsi corrigées pour l'effet des principaux facteurs du milieu (troupeau, âge, saison, durée de lactation) puis pondérées par le coefficient d'héritabilité correspondant au numéro de lactation considéré. L'information nouvelle en ce qui concerne la généalogie consiste toujours en index (index du père $I_{P}$, index de la mère $I_{M}$, index de la fille $I_{F}$ ). L'index apport est alors déduit de ces différents index. Par exemple, si l'index de la mère vient à être connu, on a $i_{\text {gen }}=\mathrm{I} / 2 \mathrm{I}_{\mathrm{M}}$. Bien entendu, dans tous les cas (performances, généalogie) on calcule le coefficient de détermination des index apports. C'est le coefficient d'héritabilité pour une nouvelle lactation 
ou un multiple (I/4) du coefficient de détermination des index des apparentés, en ce qui concerne l'index généalogie. La seconde phase de la mise à jour consiste à utiliser des formules de cumul pour l'index et son coefficient de détermination à partir des index ancien et apport et de leurs coefficients de détermination.

\section{B. - Formules de mise à jour des index et de leurs coefficients de détermination Index généalogique}

Pour établir des formules de cumul correctes convenant aux index généalogiques, il est nécessaire en théorie de distinguer si la généalogie concerne les ascendants ou les descendants.

Si l'index ancien est établi à partir de l'index du père (de la mère) et si l'indexapport est celui de la mère (du père) on montre que les formules optimales de cumul dépendent du coefficient de corrélation $p$ entre les index des parents de la manière suivante :

$$
\begin{gathered}
a_{1}=\frac{\mathrm{I}-\rho \sqrt{\frac{\mathrm{CD}_{2}}{\mathrm{CD}_{1}}}}{\mathrm{I}-\rho^{2}} \\
a_{2}=\frac{\mathrm{I}-\rho \sqrt{\frac{\mathrm{CD}_{1}}{\mathrm{CD}_{2}}}}{\mathrm{I}-\rho^{2}} \\
\mathrm{CD}=\frac{\mathrm{CD}_{1}+\mathrm{CD}_{2}-2 \rho \sqrt{\mathrm{CD}_{1} \mathrm{CD}_{2}}}{\mathrm{I}-\rho^{2}}
\end{gathered}
$$

Ces formules se déduisent des formules (I) (2) (3) (4) et (8) en faisant

car l'on a

$$
\operatorname{cov}\left(\mathrm{I}_{1}, \mathrm{I}_{2}\right)=\rho \sqrt{\mathrm{CD}_{1} \mathrm{CD}_{2}} v(\mathrm{G})
$$

$$
\begin{array}{ll}
\mathrm{I}_{1}=\mathrm{I} / 2 \mathrm{I}_{\mathrm{P}}+\varepsilon_{1} & \mathrm{CD}_{1}=\mathrm{I} / 4 \mathrm{CD}_{\mathrm{P}} \\
\mathrm{I}_{2}=\mathrm{I} / 2 \mathrm{I}_{\mathrm{M}}+\varepsilon_{2} & \mathrm{CD}_{2}=\mathrm{I} / 4 \mathrm{CD}_{\mathrm{M}}
\end{array}
$$

Quand 1'index ancien est établi à partir du père et (ou) de la mère, et quand l'index apport est établi à partir de l'index performance d'une fille, la formule de cumul peut être simplifiée si 1'on suppose qu'il n'y a pas de liaison d'origine non génétique entre l'index obtenu sur ascendance et l'index obtenu sur descendance. Cette supposition est raisonnable si l'index sur ascendance est obtenu à partir du père, utilisé en insémination artificielle et jugé sur des filles situées dans de nombreuses étables. En revanche, il peut y avoir une certaine liaison d'origine non génétique entre 1'index ascendance estimé d'après l'index de la mère et 1'index descendance, du fait qu'une vache et ses petites filles sont souvent dans la même étable et que la correction pour l'effet troupeau peut être imparfaite. En pratique, on peut négliger cette liaison, d'autant que progressivement les index ascendance seront de plus en plus des index combinés où le poids des index mère sera relativement mineur. Compte tenu de ces hypothèses, si $I_{1}$ est l'index ascendance et $I_{2} 1$ 'index descendance, on a alors $\operatorname{cov}\left(\mathrm{I}_{1}, \mathrm{I}_{2}\right)=\mathrm{CD}_{1} \mathrm{CD}_{2} v(\mathrm{G})$. Dans ce cas, les formules de cumul sont les suivantes : 


$$
\begin{aligned}
a_{1} & =\frac{\mathrm{I}-\mathrm{CD}_{2}}{\mathrm{I}-\mathrm{CD}_{1} \mathrm{CD}_{2}} \\
a_{2} & =\frac{\mathrm{I}-\mathrm{CD}_{1}}{\mathrm{I}-\mathrm{CD}_{1} \mathrm{CD}_{2}} \\
\mathrm{CD} & =\frac{\mathrm{CD}_{1}+\mathrm{CD}_{2}-{ }_{2} \mathrm{CD}_{1} \mathrm{CD}_{2}}{\mathrm{I}-\mathrm{CD}_{1} \mathrm{CD}_{2}}
\end{aligned}
$$

Les formules (9), (IO) et (II) sont identiques aux formules (I2), (I3) et (I4) si $\rho=\sqrt{\mathrm{CD}_{1} \overline{C D}_{2}}$. Les travaux de Mocouot, Flamant et Poutous (I970) permettent de penser que dans le cas des brebis laitières de race Lacaune, nous sommes à peu près dans cette situation $\left(\rho=0,20 ; \sqrt{\mathrm{CD}_{1} \mathrm{CD}_{2}}=0\right.$, Io à $\left.0, \mathrm{I} 5\right)$. Étant donné la grande analogie entre brebis et vaches laitières rencontrée jusqu'à présent en ce qui concerne les paramètres génétiques et non génétiques de variation, les formules (I2), (I3), (I4) sont utilisées en France comme seules et uniques formules de cumul des index généalogiques des femelles laitières.

\section{Index performance.}

Dans ce cas particulier, il est manifeste que la covariance entre l'ancien index $\left(\mathrm{I}_{1}\right)$ et l'index apport $\left(\mathrm{I}_{2}\right)$ est supérieure à $\mathrm{CD}_{1} \mathrm{CD}_{2} v(\mathrm{G})$, la différence provenant d'effets non génétiques liés à l'animal et non corrigés.

Si l'ancien index $I_{1}$ est un index établi sur la première lactation $P_{1}$ et si l'index $I_{2}$ est établi sur la seconde lactation $\mathrm{P}_{2}$ on a alors :

$$
\begin{aligned}
& \mathrm{I}_{1}=\mathrm{CD}_{1} \mathrm{P}_{1}=\mathrm{CD}_{1}\left(\mathrm{~A}_{1}+e_{1}\right) \\
& \mathrm{I}_{2}=\mathrm{CD}_{2} \mathrm{P}_{2}=\mathrm{CD}_{2}\left(\mathrm{~A}_{2}+e_{2}\right)
\end{aligned}
$$

où $\mathrm{CD}_{1}, \mathrm{CD}_{2}$ désignent les valeurs de l'héritabilité en première et en seconde lactation, $\mathrm{A}_{1}, \mathrm{~A}_{2}$ les valeurs correspondantes de l'effet animal ; $e_{1}, e_{2}$ les déviations aléatoires à l'effet animal. La covariance entre $I_{1}$ et $I_{2}$ passe alors de $\mathrm{CD}_{1} \mathrm{CD}_{2} \sigma_{\mathrm{G}_{1}} \sigma_{\mathrm{G}_{2}}$ à $\mathrm{CD}_{1} \mathrm{CD}_{2} \sigma_{\mathrm{A}_{1}} \sigma_{\mathrm{A}_{2}}$, en supposant qu'il existe une corrélation génétique de I entre lactations et une corrélation de I entre les effets animaux. Nous supposons que le rapport $\frac{\sigma_{\mathrm{Ai}}}{\sigma_{\mathrm{Gi}}}$ est constant et indépendant du numéro de lactation $i$. Il est égal à $\frac{r}{h^{2}}$ si l'on désigne par $r$ la part de variance due à l'effet animal (répétabilité) et $h^{2}$ le coefficient d'héritabilité. Les formules de mise à jour sont alors les suivantes :

$$
\begin{aligned}
a_{1} & =\frac{\mathrm{I}-\mathrm{CD}_{2} r / h^{2}}{\mathrm{I}-\mathrm{CD}_{1} \mathrm{CD}_{2}\left(r / h^{2}\right)^{2}} \\
a_{2} & =\frac{\sigma_{\mathrm{G}_{1}}}{\sigma_{\mathrm{G}_{2}}} \frac{\mathrm{I}-\mathrm{CD}_{1} r / h^{2}}{\mathrm{I}-\mathrm{CD}_{1} \mathrm{CD}_{2}\left(r / h^{2}\right)^{2}} \\
\mathrm{CD} & =\frac{\mathrm{CD}_{1}+\mathrm{CD}_{2}-2 \mathrm{CD}_{1} \mathrm{CD}_{2}}{\mathrm{I}-\mathrm{CD}_{1} \mathrm{CD}_{2}\left(r / h^{2}\right)^{2}} \frac{2}{\sigma_{\mathrm{G}_{1}}}
\end{aligned}
$$

Ces formules sont-elles itératives? En d'autres termes, peut-on les utiliser sans changement quand l'ancien index est lui-même issu de deux lactations 
ou plus? Si l'index ancien est obtenu à partir des deux premières lactations

$$
\left(\mathrm{I}=a_{1} \mathrm{I}_{1}+a_{2} \mathrm{I}_{2}=a_{1} \mathrm{CD}_{1} \mathrm{P}_{1}+a_{2} \mathrm{CD}_{2} \mathrm{P}_{2}\right)
$$

et si l'index apport est obtenu à partir de la troisième lactation $\mathrm{I}_{3}=\mathrm{CD}_{3} \mathrm{P}_{3}$, on en déduit que

soit

$$
\begin{aligned}
\operatorname{cov}\left(\mathrm{I}_{1} \mathrm{I}_{3}\right) & =a_{1} \mathrm{CD}_{1} \mathrm{CD}_{3} \operatorname{cov}\left(\mathrm{P}_{1} \mathrm{P}_{3}\right)+a_{2} \mathrm{CD}_{2} \mathrm{CD}_{3} \operatorname{cov}\left(\mathrm{P}_{2} \mathrm{P}_{3}\right) \\
& =\left(a_{1} \mathrm{CD}_{1} \sigma_{\mathrm{A}_{1}}+a_{2} \mathrm{CD}_{2} \sigma_{\mathrm{A}_{2}}\right) \mathrm{CD}_{3} \sigma_{\mathrm{A}_{3}} \\
& =\left(a_{1} \mathrm{CD}_{1} \sigma_{\mathrm{G}_{1}}+a_{2} \mathrm{CD}_{2} \sigma_{\mathrm{G}_{2}}\right) \mathrm{CD}_{3} \sigma_{\mathrm{G}_{3}} r / h^{2}
\end{aligned}
$$

$$
\operatorname{cov}\left(\mathrm{I}_{1} \mathrm{I}_{3}\right)=\mathrm{CDCD}_{3} \sigma_{\mathrm{G}_{1}} \sigma_{\mathrm{G}_{3}} r / h^{2}
$$

Cette formule est tout à fait analogue à celle trouvée précédemment

$$
\operatorname{cov}\left(\mathrm{I}_{1}, \mathrm{I}_{2}\right)=\mathrm{CD}_{1} \mathrm{CD}_{2} \sigma_{\mathrm{G}_{1}} \sigma_{\mathrm{G}_{2}} \frac{r}{h^{2}}
$$

Les formules (I5), I6), (I7) sont ainsi en principe utilisables quel que soit le nombre de performances ayant servi au calcul de l'index précédent. La formule optimale de calcul de $a_{2}$ (I6) est toujours la même si l'écart-type génétique ne varie pas avec le numéro de lactation ; sinon au moment du calcul de l'index $\mathrm{I}_{n+1}$ il est nécessaire de calculer le rapport des écart-types génétiques de l'index $\mathrm{I}_{n}$ et de l'index apport.

Les formules (I5), (I6), (I7), sont appliquées en France en supposant que $r / h^{2}=4 / 3$ et que l'écart-type génétique ne varie pas avec le numéro de lactation.

\section{Index global.}

L'index global est constitué à partir de l'index généalogique $\left(\mathrm{I}_{1}\right)$ et de l'index sur performances $\left(I_{2}\right)$. On peut admettre avec une bonne approximation que la covariance non génétique entre $I_{1}$ et $I_{2}$ est nulle, particulièrement si l'index $I_{1}$ fait appel à l'index du taureau père. De ce fait, les formules de cumul (I2), (I3), (I4) sont applicables.

\section{DISCUSSION DE LA MÉTHODE}

\section{A. - Avantages}

Bien que fondée sur la théorie de la régression multiple, la méthode d'indexation précédemment exposée diffère de celles habituellement rencontrées car les variables prédictrices sont, non des performances, mais déjà des index pouvant rassembler un nombre important d'informations. L'utilisation des performances entraîne toujours la constitution de matrices qui peuvent être importantes si 1'on considère les performances des ascendants de la deuxième génération (LEROY, I958; SKJERVOLD et Odehard, I959; Young, I96I ; SKJERVold, I968). Les termes de ces matrices peuvent être calculés directement à partir des répétabilités, héritabilités et corrélations génétiques. La recherche des coefficients optima des différent es sources d'information dans l'index conduit à inverser ces matrices. Il est possible alors, soit de dis- 
poser de tableaux de valeurs (coefficients des performances dans les index, coefficient de détermination des index) pour une série de situations particulières, soit de les recalculer au fur et à mesure des besoins.

\section{Capacité de stockage.}

Ces deux méthodes qui font appel à des performances supposent qu'il est possible de stocker toutes ces différentes performances ainsi que leurs précisions respectives. Or, en principe, les capacités de stockage sont souvent limitées. C'est un des avantages de la méthode que nous avons décrite de limiter à 4 le nombre d'informations à stocker par animal : 1'index généalogique et son coefficient de détermination, 1'index performance et son coefficient de détermination, l'index global pouvant se déduire facilement de ceux-ci.

\section{Mise à jour.}

La première méthode dont nous avons parlé précédemment est particulièrement limitative et difficile à mettre en œuvre pour la mise à jour. La seconde peut être particulièrement lourde au point de vue du temps de calcul si elle doit être répétée sur un grand nombre d'animaux, comme c'est le cas chez les bovins laitiers. En revanche, grâce à la méthode que nous avons décrite, il est possible d'intégrer facilement toute information nouvelle en un temps restreint, les formules de calcul ne faisant pas intervenir d'opérations matricielles.

\section{B. - Inconvénients}

La méthode de cumul des index que nous avons décrite a pour conséquence que les poids relatifs de différentes sources d'information incluses dans un index ne sont pas modifiés par l'apport de nouvelles informations. Ceci n'est en général pas le cas quand on utilise une méthode de régression multiple faisant appel à des performances et non à des index. Notre méthode peut conduire ainsi à une perte de précision.

Nous avons voulu évaluer la perte de précision obtenue dans un cas particulier qui représentera probablement à l'avenir le cas moyen rencontré dans les populations de vaches soumises au contrôle laitier, soit :
Nombre de lactations : variable
Père connu sur 50 filles
Mère connue sur 3 lactations
Grand-mère paternelle connue sur 3 lactations
Grand-père maternel connu sur 50 filles
Grand-mère maternelle connue sur 3 lactations

En supposant que $h^{2}=0,2$ et $r=0,4$, SkJERVoLd (Ig68) trouve que le coefficient de détermination de l'index global est de $0,298,0,403,0,494,0,546$ quand la vache est connue sur o, I, 3, 6 lactations, la méthode utilisée faisant appel aux performances. Quand on construit d'abord un index généalogique, un index performance puis un index combiné, les coefficients de détermination s'abaissent respectivement à 0,$298 ; 0,403 ; 0,48 \mathrm{I} ; 0,522$. La chute de précision s'accroît bien entendu avec le nombre de performances, car l'augmentation de la connaissance des per- 
formances de l'individu conduit en théorie à faire varier l'intérêt relatif des performances des différents apparentés (par exemple le poids de la performance de la grand-mère paternelle diminue plus vite que celui des performances des filles du père), alors que selon notre méthode, cet intérêt relatif est constant. On peut constater cependant qu'au bout de 6 lactations, la perte de précision n'est que de $5 \mathrm{p}$. Ioo.

Dans l'exemple précédent, on a supposé que la valeur de l'héritabilité était constante. Dans ce cas, on constate que notre méthode ne conduit à aucune perte de précision sur l'index performance, car les rapports entre les poids optima des différentes lactations sont constants. Or, dans les calculs pratiques d'index on a supposé (Poutous et Crochez, I97I) que $h^{2}=0,3$ en Ire lactation et $h^{2}=0,2 \mathrm{I}$ pour les suivantes, avec $\frac{r}{h^{2}}=\frac{4}{3}$. La perte de précision interviendrait donc à partir de la troisième lactation, car pour la seconde lactation, l'index cumul est en fait constitué à partir d'une seule lactation. En fait, l'étude théorique montre qu'il n'y a aucune perte de précision si la variance génétique, et non le coefficient dthéritabilité, est constante avec le numéro de lactation. ForsTER (I97I) en étudiant l'intérêt d'une formule approximative d'index trouve une perte de précision de $0,4 \mathrm{p}$. Ioo en troisième lactation, de $\mathrm{I}, 6 \mathrm{p}$. Ioo en $5^{\mathrm{e}}$ lactation quand les coefficients d'héritabilité sont de 0,30 en première lactation, 0,24 en seconde lactation et 0,20 pour les lactations ultérieures, mais dans ce cas, la variance génétique n'est pas considérée comme constante. Les variations d'héritabilité, du moins celles habituellement considérées dans le cas des bovins laitiers et associées à des variations d'écart-type génétique, ne sont donc pas une cause importante d'erreur pour la méthode simplifiée de calcul d'index.

Reçu pour publication en novembre 1972.

\section{SUMMMARY}

\section{METHODS OF COMPUTING THE MILK PRODUCTION INDEX OF FEMALES IN FRANCE}

The paper describes the methods of computing and up-dating cow indices currently used for selection in France.

The information available up to a given time (ancestors, own performance or progeny, is summarized in an index to which a determination coefficient may be attached, which measures its accuracy. In adding supplementary information, the previously computed index is used without reference to the basic data from which it was calculated. Little or no accuracy is lost by this procedure which allows a reduction in the amount of data stored and a simplification of the formulae used in calculation, important factors in using such indices in large populations.

\section{RÉFÉRENCES BIBLIOGRAPHIQUES}

FöRSTER F., I971. Zuchwertschätzung von Kühen auf Grund der Leistungen mehrever Laktationen. Diss. Hohenheim.

Le Roy H. L., 1958. Die Abstammungsbewertung. Z. Tierziucht Züchtbiol., r1, 328-278.

Lindme B., I968. Model simulation of AI breeding within a dual purpose breed of cattle. Acta Agr. Scand., 18, $33-4 \mathrm{I}$. 
LINDSTRÖM U., I969. Genetic change in milk yield and fat percentage in artificially bred populations of finnish dairy cattle. Acta Agr. Fenn., 114, I-128.

Mocouot J.-C., Flamant J.-C., Poutous M., r97o. Aspects quantitatifs de la production laitière des brebis. VI. Relations entre les index laitiers sur ascendance et sur descendance des reproducteurs mâles de race Lacaune. Ann. Génét. Sél. anim., 2, 53-63.

Poutous M., Crochez S., x97r. Méthode de calcul des index de production laitière. V Index laitiers des femelles. Bull. Tech. Ing. Serv. Agric., (263), 855-861.

Skjervold H., I968. Kombinierte Selektion. Acta Agr. Scand., 18, 65-79.

SKJERVOLD H., ODEGARD A. K., I959. Estimation of breeding value on the basis of the individual's own phenotype and ancestor's merit. Acta. Agr. Scand., 9, 34I-354.

SKJERVold H., LANGHolz H. J., 1964. Factors affecting the optimum structure of AI breeding in dairy cattle. Z. Tierziücht. ZiüchtBiol, $80,25-40$.

Syrstad O., I966. Studies on dairy herd records. III. Estimation of genetic change. Acta. Agr. Scand., $16,3-14$.

Young S. S. Y., 196r. The use of sire's and dam's records in animal selection. Heredity, 16, 9r-102. 